Volume 8, No.1.6, 2019

International Journal of Advanced Trends in Computer Science and Engineering

Available Online at http://www.warse.org/IJATCSE/static/pdf/file/ijatcse0181.62019.pdf

https://doi.org/10.30534/ijatcse/2019/0181.62019

\title{
Measurement of Different Illumination and Image Target on Complementary Metal Oxide Semiconductor (CMOS)
}

\author{
Norsuzila Ya'acob $^{1,2}$, Noraisyah Tajudin', Rabi'atul Addawiyah Zulkiplee ${ }^{1}$, Azita Laily Yusoff ${ }^{1,2}$, Suzi Seroja Sarnin ${ }^{1,2}$, \\ Murizah Kassim ${ }^{1}$ and Nani Fadzlina Naim ${ }^{1}$ \\ ${ }^{1}$ Faculty of Electrical Engineering,Universiti Teknologi MARA (UiTM) Malaysia,40450 Shah Alam, Selangor,Malaysia. \\ ${ }^{2}$ Wireless Communication Technology (WiCoT), Faculty of Electrical Engineering, Universiti Teknologi MARA, 40450 Shah \\ Alam Selangor, Malaysia \\ norsuzila@uitm.edu.my, tnoraisyah@gmail.com,addawiyah.zulkiplee@gmail.com, azita968@ uitm.edu.my, \\ suzis045@uitm.edu.my, murizah@uitm.edu.my,nanifadzlina@uitm.edu.my
}

\begin{abstract}
This research is about the Measurement of Different Types of Illumination and Image Target on Complementary Metal Oxide Semiconductor (CMOS). The problem for this research is the sensitivity of the CMOS and the resistance of CMOS toward blooming and smearing. The objective of this research is to measure the spectral sensitivity and the pixel intensity of the output image and to compare the spectral sensitivity and pixel intensity using different illumination and prime lens with different diameters. This project used CMOS camera and two different target images to measure the spectral sensitivity of every image and the outcomes of digital value on pixel peek of the CMOS. Thor Cam software is used to measure the pixel intensity of the image and histogram graph for spectral sensitivity. Different diameter of a prime lens is used, $12 \mathrm{~mm}$ and $16 \mathrm{~mm}$. The illumination and the diameter of the prime lens effect the sensitivity, output of spectral sensitivity and pixel intensity.
\end{abstract}

Key words: Image processing, RGB, pixel intensity, prime lens

\section{INTRODUCTION}

Image processing is an analysis and manipulation of a digitized image, especially in order to improve the quality. Changing CMOS circuits would do well to execution and need less silicon zone over traditional static CMOS circuits [1]. With the evolution of multimedia technology, the usage of large image database has rapidly increased [2]. The remarkable advances in complementary metal-oxidesemiconductor (CMOS) process technology, a variety of vision sensors with signal processing circuits for complicated functions are actively being developed [3]. Image processing is a method to perform some operations on an image in order to get an enhanced image processing a method to perform some operations on an image or to extract some useful information from it. It is a type of signal processing in which input is an image and output may be image or characteristics or features associated with that image or to extract some useful information from it.

The proposed method segments the whole image into several regions and calculates the local histograms for each region in order to achieve localization, giving more accurate results [4, 5]. The effectiveness of the system can be significantly improved by restricting the search to the images that are alike to the query image $[6,7]$. Any image can be well described by an image descriptor which utilizes some of the features of the image to do so. These features include color, texture, shape etc. The color histogram, which defines the distribution of different intensity levels of color in an image, is used as an image descriptor in this paper [8]. CMOS will be used as the main subject to analyze several data from it.

According to Utsav Jain [9] state that CMOS Image Sensors is semiconductor device used for making a digital camera. CMOS Image Sensor detect information in the form of light or any other electromagnetic radiation and create an image that represents the information. CMOS image sensors consist of integrated circuits that sense the information and convert it into an equivalent current or voltage which is later converted into digital data.

CMOS camera sensors are much less expensive to manufacture. Ann Holms [10] state that the overall system of CMOS only consuming small and less power because all the required circuitry and components already integrated onto the sensor. CMOS filter will convert light (photon) into electron and then convert the electron into voltage and project an image from it. M. Bigas [11] state that with the improvement of CMOS camera sensor a new application sensor is being opened up such as space application and medical.

Buddola Viswanath [12] state that the latest development of CMOS image sensor is in biomedical research and diagnosis. This is due to the CMOS technology that are low cost, lower power is required, manufacturable and also consistent. 
O.Saint Pe' [13] state that the main space application is optical payload and sensor. CMOS image sensor is being used widely in space application due to faster access to information, better radiation behavior and also low power consumption. Two types of CMOS camera sensor is being used in this research, CMOS Color Camera and CMOS Monochrome Camera. The CMOS Color camera give a output of pixel in RED, BLUE, GREEN pixel while the CMOS Monochrome camera will give a output in mono.

Illumination or light definition by Paul E.Tippens [14] state that light may be thought of as a little bundles of wave emitted in discrete packet called photon. In this research the effect of light condition that being use is being observe to see the effect on CMOS camera. The objective of this research is about to measure the spectral sensitivity and the pixel intensity of the output image and to compare the spectral sensitivity and pixel intensity using different illumination and prime lens with different diameter.

\section{METHODOLOGY}

In this research a several measurement are done in order to get the spectral sensitivity and the outcomes of digital value from the target image.

\subsection{Material}

i) Complementary Metal Oxide Semiconductor (CMOS) These CMOS cameras have two types of it, CMOS Color and CMOS Monochrome. This CMOS works by convert the light or photon into electron and then it will generate image $[15,16,17,18]$.

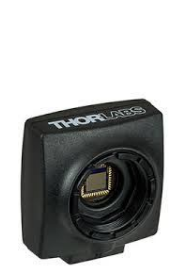

(a) CMOS

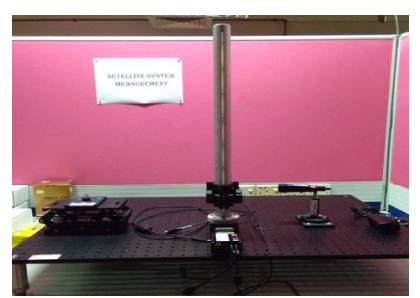

(b) Optic table
Figure 1: (a) CMOS and (b) Optic table To assessing perceptual image quality traditionally attempted to quantify the visibility of errors (differences) between a distorted image and a reference image using a variety of known properties of the human visual system. The method use in this research is by introducing an alternative complementary framework for quality assessment based on the degradation of structural information [19,20,21,22,23].

iii) ThorCam Software

By using this ThorCam software, the spectral sensitivity from histogram graph and outcomes of digital value of target image will be obtain.

\subsection{Method}

For the measurement two type of CMOS camera is being used, CMOS Color camera and CMOS Monochrome camera. Classification [24] is the process of classifying instances into their respective classes. Classification comprises of variables with known values to predict the unknown or future values of other variables. This CMOS camera will be measure it sensitivity while capture a image under two different light source condition and using two different prime lens with diameter $12 \mathrm{~mm}$ and $16 \mathrm{~mm}$.

\section{i) Flowchart}

Based on Figure 2 the flowchart, firstly the equipment is being setup by installation of CMOS camera and connect the CMOS camera to the computer for ThorCam software to detect the CMOS camera. Two different light source surrounding light and fiber optic and also two different prime lens with diameter $12 \mathrm{~mm}$ and $16 \mathrm{~mm}$ is being used.

After that the target image is being installed on the actuator in front of the CMOS camera. Before capturing the image target the adjustment of lens is being made to ensure the target image is in focus and clear. Thinku Acharya [25] state that if the camera is not appropriately focused a blurred image will be obtain. The output of the image targets that being capture will be obtained from the THORLAB software. The histogram graph and pixel peek will be obtained from the image that had been capture.

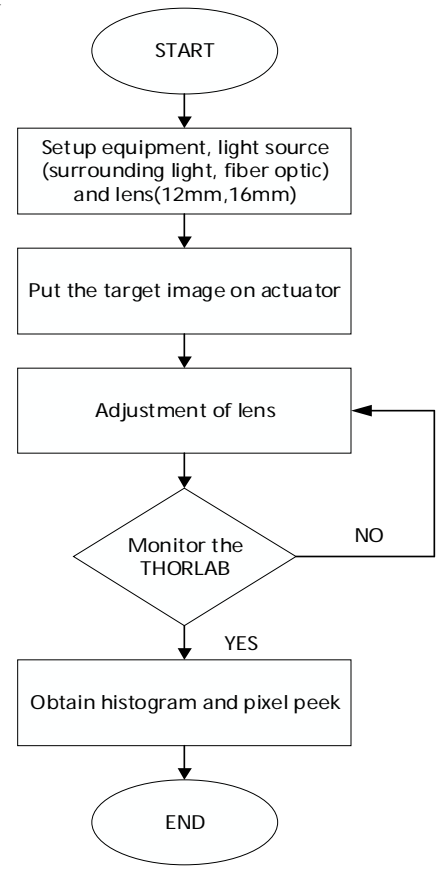

Figure 2: Flowchart 


\section{RESULT AND DISCUSSION}

\subsection{Measurement of CMOS Color camera with $12 \mathrm{~mm}$ and $16 \mathrm{~mm}$ prime lens and using surrounding light and fiber optic}

\section{i) $12 \mathrm{~mm}$ prime lens with surrounding light and fiber optic}

Figure 3 is material 1 with a transparent background and material 2 with a black background. This image target is made up of glass. This image target is being captured under a surrounding light condition using a prime lens with a diameter of $12 \mathrm{~mm}$. When using the surrounding light the image looks more clear and focus. The detail printed on the material can be seen. As material 2 using a black background the image looks a little darker than the image of material 1 that has a transparent background.

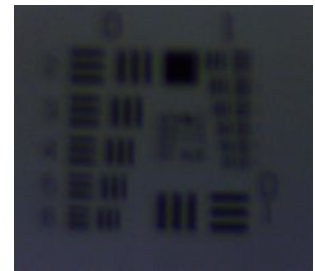

(a) Material 1

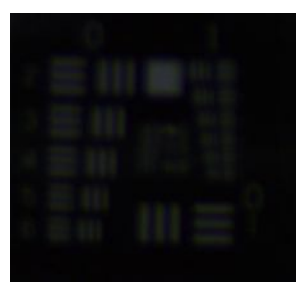

(b) Material 2

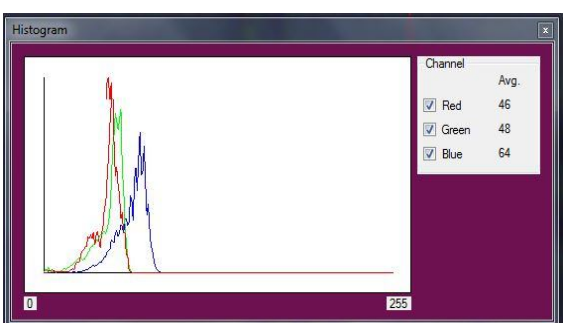

(c) Histogram of material 1

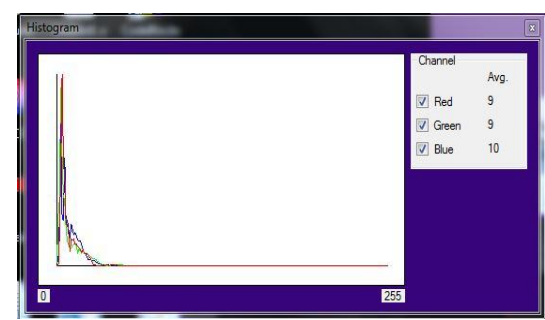

(c) Histogram of material 2.

Figure 3: (a) Material 1 with transparent background, (b) Material 2 with black background, (c) histogram of material 1 and (d) histogram of material 2.

Figure 3 (c) and (d) shows that the spectral sensitivity of material 1 is measured. When using CMOS Color camera, the spectral sensitivity will divide into three red, blue and green. The red, blue and green is represented the pixel intensity for material 1 and material 2. At Figure 3 (c) the spectral sensitivity shows the measurement of red, blue, green pixel. From the spectral sensitivity, the red pixel is the highest following with green and blue. That means from the image of material 1 pixel of red being captured more other than blue and green. Figure 3 (d) the spectral sensitivity of material 2 is measure. The spectral sensitivity is near to 0 scale. This thing happen due to the image is darker.

Figure 4 is the (a) material 1 with a transparent background and (b) material 2 with a black background. This image target is made up of glass. Both of the image targets are being captured under a light source from a fiber optic using a prime lens with diameter $12 \mathrm{~mm}$. since using the fiber optic as the light source both material is exposed under a low light condition. Due to this situation, a black image becomes the outcome of the image output. In material 1 image a small amount of light can be seen while in material 2 images is completely dark this happens due to the black background of the material 2. Light from the fiber optic cannot pass through the material 2 .

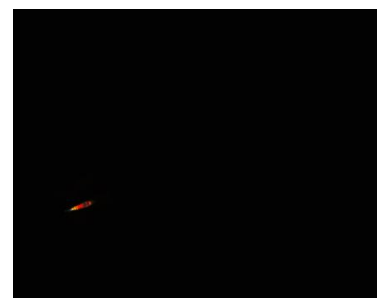

(a) Material 1

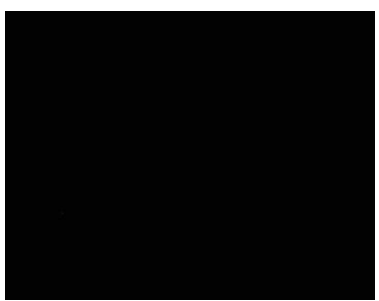

(b) Material 2

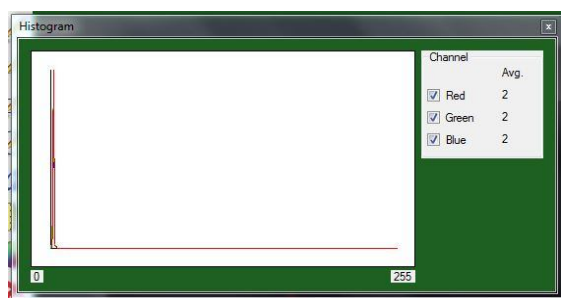

(c) Histogram of material 1

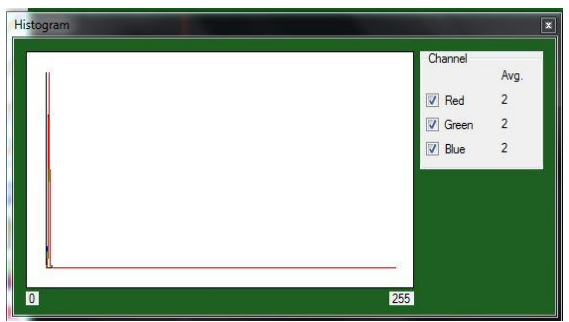

(d) Histogram of material 2

Figure 4: (a) Material 1 with transparent background, (b) Material 2 with black background, (c) Histogram of material 1 and (d) Histogram of material 2.

Figure 4 (c) and (d) shows that the spectral sensitivity of material 1 and material 2 are measured. When using CMOS Color camera, the spectral sensitivity will divide into three red, blue and green. The red, blue and green are represented the pixel intensity for material 1 and material 2. Because the image target is under low light condition due to the light source from fiber optic small amount of light is capture 
make the spectral sensitivity is more to 0 due to low pixel is being captured and the image is almost saturated black.

\section{ii) $16 \mathrm{~mm}$ prime lens with surrounding light and fiber optic}

Figure 5 is (a) material 1 with a transparent background and (b) material 2 with a black background. This image target is made up of glass. This image target is being captured under a surrounding light condition using a prime lens with a diameter of $16 \mathrm{~mm}$. The image of material 1 and material 2 look more clear and focus under the light conditions of surrounding light.

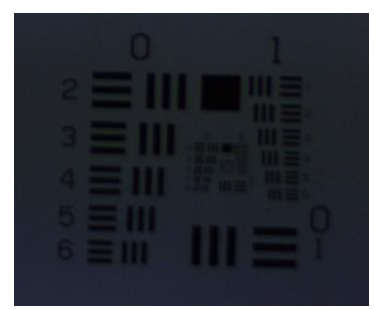

(a) Material 1

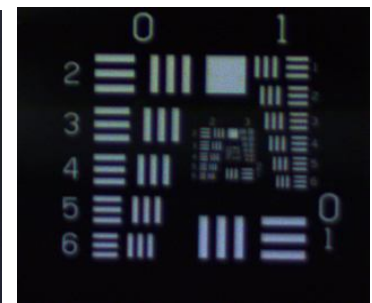

(b) Material 2

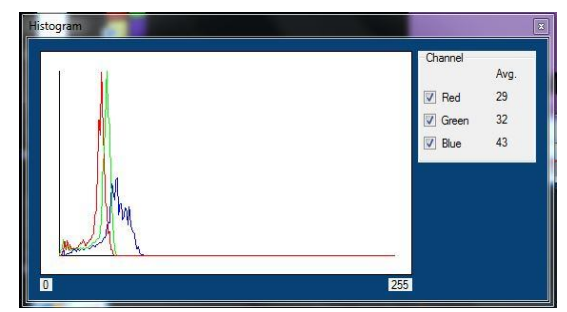

(e) Histogram of material 1

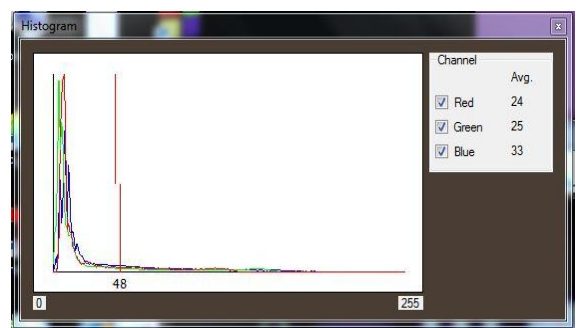

(f) Histogram of material 2

Figure 5: (a) Material 1 with transparent background, (b) Material 2 with black background, (e) Histogram of material 1 and (f) histogram of material 2.

According to Figure 5 (e) and (f), the spectral sensitivity of material 1 and material 2 are measured. As the figure above when using CMOS Color camera, spectral sensitivity will divide into three red, blue and green. The red, blue and green are represented the pixel intensity for material 1 and material 2. Due to the black background of the histogram of material 1 , spectral sensitivity is near to 0 pixels.

Figure 6 is (a) material 1 with a transparent background and (b) material 2 with a black background. This image target is being captured under a light source from a fiber optic using a prime lens with a diameter of $16 \mathrm{~mm}$. While using fiber optic as the light source, the materials are under low light conditions. Due to this situation, only a small amount of light can be captured from CMOS camera and make the images look dark.

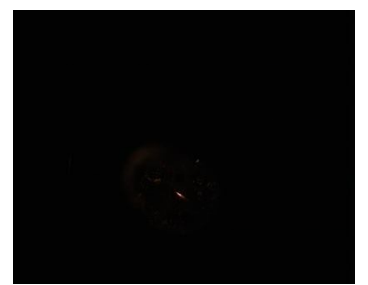

(a) Material 1

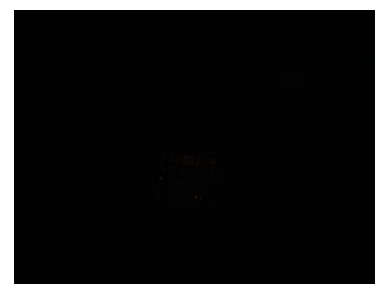

(b) Material 2

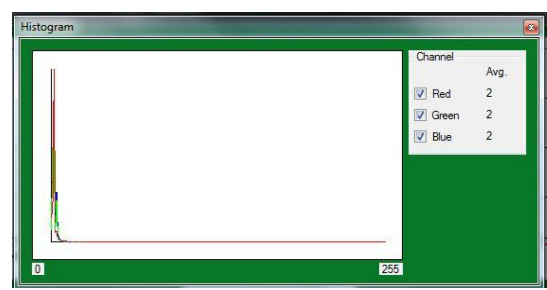

(c) Histogram of material 1

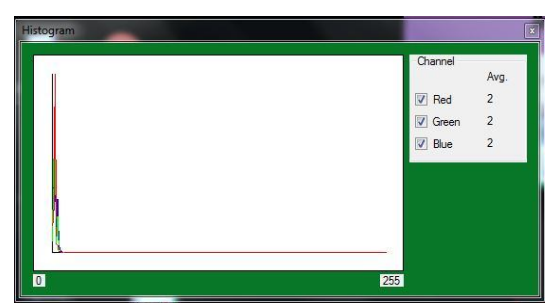

(d) Histogram of material 2

Figure 6: (a) Material 1 with transparent background, (b) Material 2 with black background, (c) Histogram of material 1 and (d) Histogram of material 2.

Figure 6 (c) and (d) shows that the spectral sensitivity of material 1 and material 2 are measured. When using CMOS Color camera the spectral sensitivity will divide into three red, blue and green. The red, blue and green are represented the pixel intensity for material 1 and material 2. Because the image target is under low light condition due to the light source from the fiber-optic small amount of light is capture make the spectral sensitivity is more to 0 due to low pixel is being captured and the image is almost saturated black. The same thing happens as (d).

\subsection{Measurement of CMOS Monochrome camera with $12 \mathrm{~mm}$ and $16 \mathrm{~mm}$ prime lens and using surrounding light and fiber optic}

\section{i) $12 \mathrm{~mm}$ prime lens with surrounding light and fiber optic}

Figure 7 is (a) material 1 with a transparent background and (b) material 2 with a black background. This image target is 
made up of glass. This image target is being captured under a surrounding light condition using a prime lens with a diameter of $12 \mathrm{~mm}$ using CMOS Monochrome. The image looks clear and focuses due to the surrounding light condition.

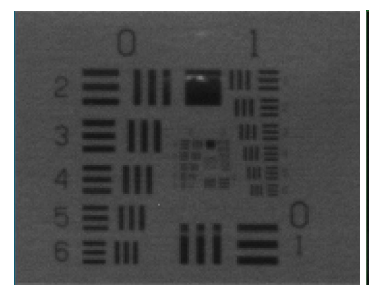

(a) Material 1

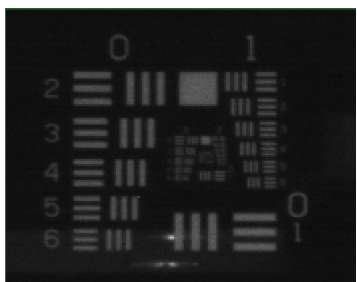

(b) Material 2

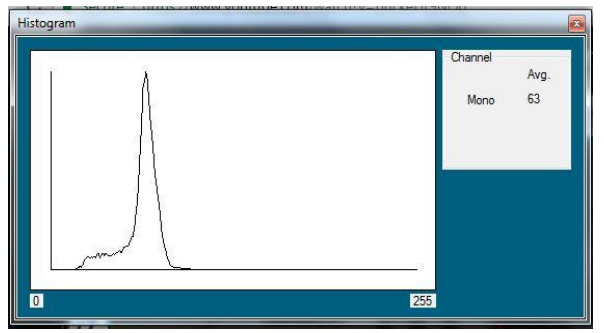

(c) Histogram of material 1

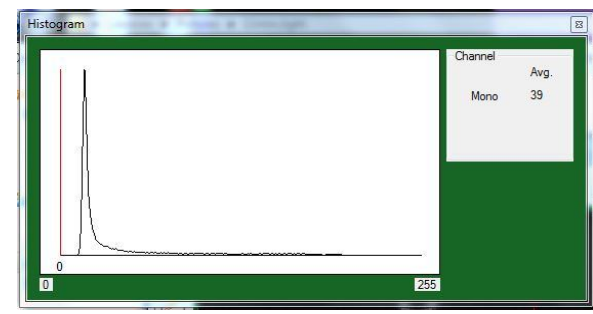

(d) Histogram of material 2

Figure 7: (a) Material 1 with transparent background and (b) Material 2 with black background (c) Histogram of material 1 and (d) Histogram of material 2.

According to Figure 7 (c) and (d), the spectral sensitivity of material 1 and material 2 are measured. When using CMOS Monochrome camera the spectral sensitivity will be in mono. The mono is represented as the pixel intensity for material 1and material 2. In (d) the spectral sensitivity is more to 0 pixels. This happens due to the background of material 2 is black. The image looks darker than image of material 1.

Figure 8 is (a) material 1 with a transparent background and (b) material 2 with a black background. This image target is made up of glass. This image target is being captured under a light source from a fiber optic using a prime lens with a diameter of $12 \mathrm{~mm}$ using CMOS Monochrome camera. CMOS Monochrome camera captured lighter under low light conditions. As can be seen the amount of light in the image for both materials are more compare when using CMOS Color camera.

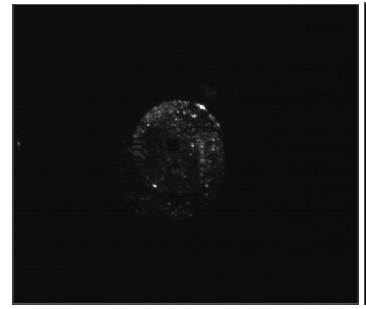

(a) Material 1

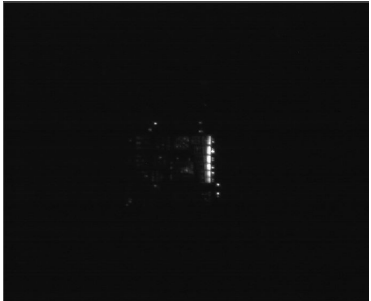

(b) Material 2

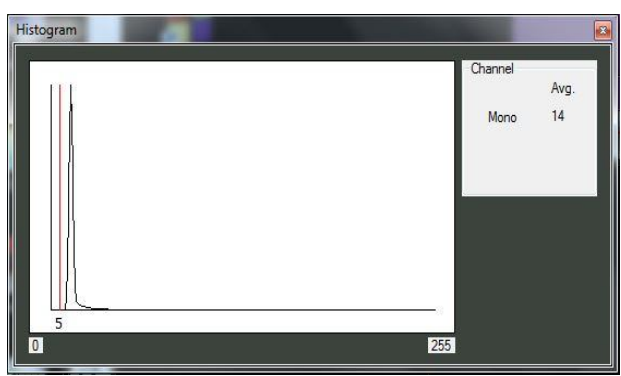

(c) Histogram of material 1

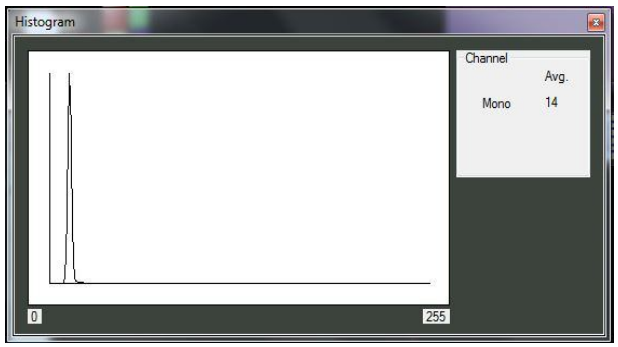

(d) Histogram of material 2

Figure 8: (a) Material 1 with transparent background, (b) Material 2 with black background, (c) Histogram of material 1 and (d) Histogram of material 2.

Figure 8 (c) and (d) shows the spectral sensitivity of material 1and material 2 are measured. As the figure 8 above when using CMOS Monochrome camera, spectral sensitivity will be in mono. The mono is represented as the pixel intensity for material 1 and material 2. Due to low light condition when placing the image and using fiber optic as the light source, the spectral sensitivity is near to 0 pixel cause the image is almost saturated black.

\section{ii) $16 \mathrm{~mm}$ prime lens with surrounding light and fiber optic}

Figure 9 (a) and (b) which are Figure 9 (a) material 1 with transparent background and Figure 9 (b) material 2 with a black background. This image target is made up of glass. This image target is being captured under a light source from a surrounding light using a prime lens with a diameter of $16 \mathrm{~mm}$ using CMOS Monochrome camera. Under light condition using surrounding light the image target looks more clear and focus. The material 2 look darker than material 1 due to the background of the material 2 . 


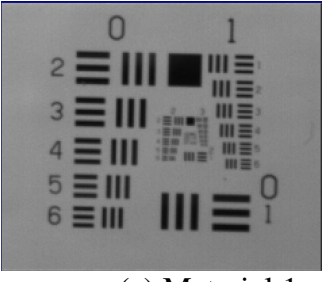

(a) Material 1

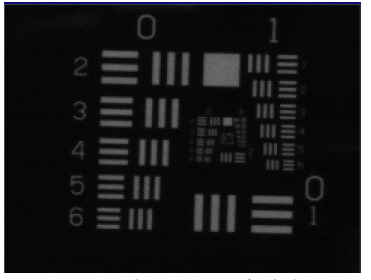

(b) Material 2

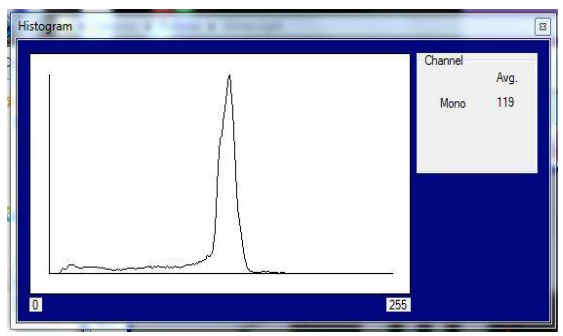

(c) Histogram of material 1

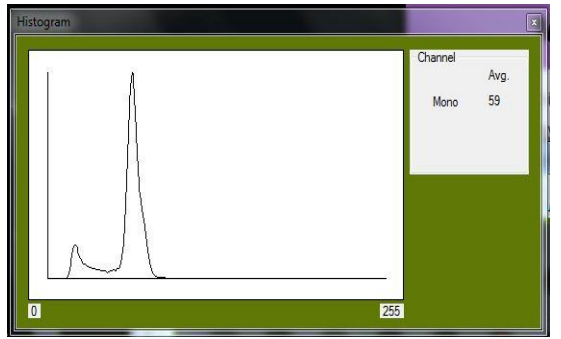

(d) Histogram of material 2

Figure 9: (a) Material 1 with transparent background, (b) Material 2 with black background, (c) Histogram of material 1 and (d) Histogram of material 2.

Figure 9 (c) and (d) shows that the spectral sensitivity of material 1 and material 2 are measured. When using CMOS Monochrome camera the spectral sensitivity will be in mono. The mono is represented as the pixel intensity for material 1 and material 2. CMOS Monochrome camera captured more pixels under the light condition of the surrounding light. The average pixel intensity is higher when using the surrounding light.

Figure 10 (a) and (b) which are (a) Material 1 with a transparent background and (b) Material 2 with a black background. This image target is made up of glass. This image target is being captured under a light source from a fiber optic using a prime lens with a diameter of $16 \mathrm{~mm}$ using CMOS Monochrome Camera. Due to low light conditions, only a small amount of light is being captured and the image looks darker compare when using surrounding light.

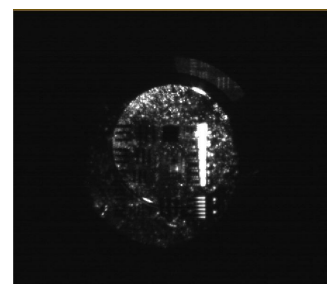

(a) Material 1

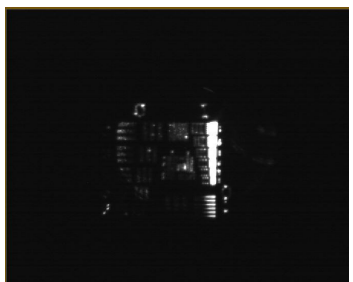

(b) Material 2

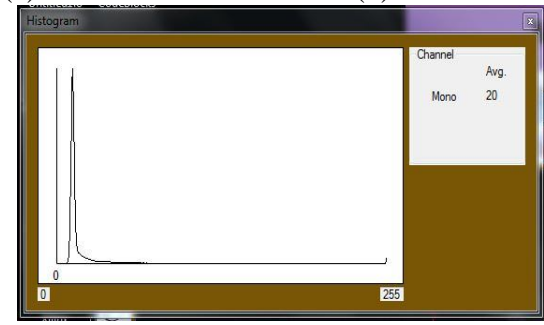

(c) Histogram of material 1

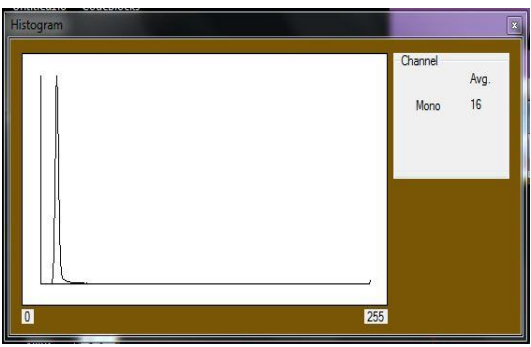

(d) Histogram of material 2

Figure 10: (a) Material 1 with transparent background, (b) Material 2 with black background, (c) Histogram of material 1 and (d) Histogram of material 2.

Figure 10 (c) and (d) shows that the spectral sensitivity of material 1 and material 2 are measures. When using CMOS Monochrome camera, the spectral sensitivity will be in mono. The mono is represented as the pixel intensity for material 1 and material 2. When a dark image is being captured the spectral sensitivity will near to 0 pixels due to image almost saturated black. Under the low light conditions, the average pixel intensity is low compare to pixel intensity when using the surrounding light.

\subsection{Comparison}

i) Comparison between $12 \mathrm{~mm}$ and $16 \mathrm{~mm}$ prime lens by using CMOS color.

Table 1: Comparison between $12 \mathrm{~mm}$ and $16 \mathrm{~mm}$ prime lens by using CMOS color.

\begin{tabular}{|l|l|l|l|l|}
\hline $\begin{array}{c}\text { Type of light } \\
\begin{array}{c}\text { source/ types of } \\
\text { prime lens }\end{array}\end{array}$ & \multicolumn{2}{|c|}{$\begin{array}{c}12 \mathrm{~mm} \\
\text { (pixel intensity) }\end{array}$} & \multicolumn{2}{c|}{$\begin{array}{c}16 \mathrm{~mm} \\
\text { (pixel intensity) }\end{array}$} \\
\cline { 2 - 5 } & $\begin{array}{c}\text { Material } \\
1\end{array}$ & $\begin{array}{c}\text { Material } \\
2\end{array}$ & $\begin{array}{c}\text { Material } \\
1\end{array}$ & $\begin{array}{c}\text { Material } \\
2\end{array}$ \\
\hline Surrounding light & $\mathrm{R}: 46$ & $\mathrm{R}: 9$ & $\mathrm{R}: 29$ & $\mathrm{R}: 24$ \\
& $\mathrm{G}: 48$ & $\mathrm{G}: 9$ & $\mathrm{G}: 32$ & $\mathrm{G}: 25$ \\
& $\mathrm{~B}: 64$ & $\mathrm{~B}: 10$ & $\mathrm{~B}: 43$ & $\mathrm{~B}: 33$ \\
\hline Fiber optic & $\mathrm{R}: 2$ & $\mathrm{R}: 2$ & $\mathrm{R}: 2$ & $\mathrm{R}: 2$ \\
& $\mathrm{G}: 2$ & $\mathrm{G}: 2$ & $\mathrm{G}: 2$ & $\mathrm{G}: 2$ \\
& $\mathrm{~B}: 2$ & $\mathrm{~B}: 2$ & $\mathrm{~B}: 2$ & $\mathrm{~B}: 2$ \\
\hline
\end{tabular}


From Table I the value of outcomes digital value of a pixel in average from Red, Blue, Green can be obtained. From the average value of pixel intensity. For CMOS camera that used a prime lens with a diameter of $12 \mathrm{~mm}$, the value of the average intensity is smaller than the average pixel intensity with the CMOS camera that used a prime lens with a $16 \mathrm{~mm}$ diameter. This is due to the higher the exposure of the lens toward target image, the higher the amount of digital value being gather and also the aperture of the lens will be higher and make the CMOS camera with prime lens $16 \mathrm{~mm}$ diameter capture lighter into CMOS.

But due to material 2 background is black the amount of light that passes through and being captured with CMOS camera is low compare with material 1 with transparent background. As to be seen the amount of pixel intensity for material 2 is low compared to material 1 even when using surrounding light. When different illumination types being used, surrounding light and fiber optic, the CMOS camera capture more amount of digital value in surrounding light than fiber optic. This is due to when using fiber optic CMOS camera will capture under the low light condition and the amount of light signal gather into CMOS is also low.

ii) Comparison between $12 \mathrm{~mm}$ and $16 \mathrm{~mm}$ prime lens by using CMOS monochrome

Table 2: Comparison between $12 \mathrm{~mm}$ and $16 \mathrm{~mm}$ prime lens by using CMOS monochrome

\begin{tabular}{|l|c|c|c|c|}
\hline $\begin{array}{c}\text { Type of light } \begin{array}{c}\text { source/ types of } \\
\text { prime lens }\end{array} \\
\end{array}$ & \multicolumn{2}{|c|}{$\begin{array}{c}12 \mathrm{~mm} \\
\text { (pixel intensity) }\end{array}$} & \multicolumn{2}{c|}{$\begin{array}{c}16 \mathrm{~mm} \\
\text { (pixel intensity) }\end{array}$} \\
\cline { 2 - 5 } & $\begin{array}{c}\text { Material } \\
1\end{array}$ & $\begin{array}{c}\text { Material } \\
2\end{array}$ & $\begin{array}{c}\text { Material } \\
1\end{array}$ & $\begin{array}{c}\text { Material } \\
2\end{array}$ \\
\hline Surrounding light & 63 & 39 & 119 & 59 \\
\hline Fiber optic & 14 & 14 & 20 & 16 \\
\hline
\end{tabular}

From table II by using CMOS monochrome with $12 \mathrm{~mm}$ and $16 \mathrm{~mm}$ diameter of the prime lens the amount of the digital value from pixel peek and also can be obtained. When using the CMOS camera with a prime lens $12 \mathrm{~mm}$ diameter the amount of average pixel intensity is lower than CMOS camera with $16 \mathrm{~mm}$ diameter. When different illumination types being used, surrounding light and fiber optic, the CMOS monochrome camera capture more amount of digital value in surrounding light than fiber optic. This is due to when using fiber optic CMOS camera will capture under the low light condition and the amount of signal light gather in CMOS is also low. This result is the same with the CMOS color camera..

\section{CONCLUSION}

From the research, the comparison of different illumination and material on CMOS, two different CMOS cameras are being used, CMOS color camera and CMOS monochrome camera. These two types of CMOS camera used two different lenses with different diameter, $12 \mathrm{~mm}$ and $16 \mathrm{~mm}$. When the amount of digital value and spectral sensitivity need the observe the camera is located under two different situations of light condition, surrounding light, and fiber optic. The amount of digital value of pixel peek is higher when both CMOS camera using a prime lens with $16 \mathrm{~mm}$ diameter and using the surrounding light as the light source. This is due to the wider the aperture of lens more digital value will be gathered from the target image. But the material background might be effect the pixel intensity value because the image may look darker than material 1 .

\section{ACKNOWLEDGMENT}

The authors would like to thank Faculty of Electrical Engineering, Universiti Teknologi MARA (UiTM) for their valuable support. This research is partly funded by the Malaysian Government through UiTM under 600RMI/DANA5/3/GIP (042/2019).

\section{REFERENCES}

1. D G Sankar Rao,N.M.Ramalingeshwar, D.Vijendra Kumar, Simhadri Kollu, International Journal of Advanced Trends in Computer Science and Engineering, Vol. 7, No. 6, pp. 99-102, Dec. 2018.

2. D. Sarala, Tejas Kanikdaley, Sharad Jogi, Rahul K. Chaurasiya, Content-Based Image Retrieval Using Hierarchical Color and Texture Similarity Calculation, International Journal of Advanced Trends in Computer Science and Engineering, Vol. 7, No. 2,PP. 11-16, April 2018. https://doi.org/10.30534/ijatcse/2018/02722018

3. Jong-Ho Park, Jung-Hwan Kim, Sung-Ho Suh, JangKyoo Shin, Minho Lee, Pyung Choi and Tetsuya Yagi, A Complementary Metal-Oxide-Semiconductor Vision Chipfor Edge and Motion Detection with a Functionfor Output Offset Cancellation, Optical Review, Vol. 12, No. 1, pp. 15-19, 2005.

https://doi.org/10.1007/s10043-005-0015-0

4. N. Shrivastava and V. Tyagi. Content based image retrieval based on relative locations of multiple regions of interest using selective regions matching, Information Sciences, vol. 259, pp. 212-224, 2014.https://doi.org/10.1016/j.ins.2013.08.0436.

5. M. E. ElAlami, A novel image retrieval model based on the most relevant features, Knowledge-Based Syst., vol. 24, no. 1, 2011. 7.

6. S. Youssef. ICTEDCT-CBIR: Integrating curvelet transform with enhanced dominant colorsextraction and texture analysis for efficient content-based image retrieval, Computers \& Electrical Engineering, vol. 38, no. 5, pp. 1358-1376, 2012.https://doi.org/10.1016/j.compeleceng.2012.05.01 08 .

7. Simardeep Kaur, Vijay Kumar Banga. Content Based Image Retrieval: Survey and Comparison between RGB and HSV model, International Journal of Engineering Trends and Technology (IJETT), vol. 4, no. 4, April 2013.

8. D. Sarala, Tejas Kanikdaley, Sharad Jogi, Rahul K. Chaurasiya, Content-Based Image Retrieval Using Hierarchical Color and Texture Similarity Calculation, International Journal of Advanced Trends in Computer Science and Engineering, Vol. 7, No. 2, pp. 11-16, April 2018. 
https://doi.org/10.30534/ijatcse/2018/02722018

9. Utsav Jain, Characterization of CMOS Image Sensor, Master of Science Thesis, 2016.

10. H.Ann and Q.Alvin., Complementary Metal Oxide Semiconductor Sensors, 2010.

11. M. Bigasa, E. Cabrujaa, J. Forestb , J.Salvi, Review of CMOS image sensors, Microelectronics Journal 37,pg 433-451,2006 https://doi.org/10.1016/j.mejo.2005.07.002

12. Buddolla Viswanath, Yuzon Ma Kristine and Sanghyo Kim, Recent trends in the development of complementary metal oxide

13. semiconductor image sensor to detetct foodborne bacteria pathogens, Trend in Analytical Chemistry 98,pg 47-57, 2018

https://doi.org/10.1016/j.trac.2017.10.019

14. Mohsen Farhadloo, Erik Rolland, Multi-Class Sentiment Analysis with Clustering and Score Representation, IEEE 13th International Conference on Data Mining Workshops, pp. 904-912, 2013. https://doi.org/10.1109/ICDMW.2013.63

15. Nan Chen, Shengyou Zhong, Mei Zou, Jiqing Zhang, Zhongshun Ji, and Libin Yao, Senior Member and IEEE , A Low-Noise CMOS Image Sensor With Digital Correlated Multiple Sampling, IEEE Transactions On Circuits And Systems-I: Regular Papers, 2017. https://doi.org/10.1109/TCSI.2017.2724855

16. Denny Lie, Kwanyeob Chae, and Saibal Mukhopadhyay, Analysis of the Performance, Power, and Noise Characteristics of a CMOS Image Sensor With 3-D Integrated Image Compression Unit, IEEE Transactions On Components, Packaging And Manufacturing Technology, Vol. 4, No. 2, February 2014. https://doi.org/10.1109/TCPMT.2013.2295412

17. Hae-Gon Jeo, Joon-Young Lee,Sunghoon Im, Hyowon $\mathrm{Ha}$, and In So Kweon, Stereo Matching with Colorand Monochrome Camerasin Low-light Conditions, IEEE Conference on Computer Vision and Pattern Recognition,2016.

18. Elena S. Yelmanova and Yuriy M. Romanyshyn, Definitions of Weighted and Relative Contrasts of Elements of Monochrome Images, CSIT 2017 https://doi.org/10.1109/STC-CSIT.2017.8098810.

19. Zhou Wang, Member, IEEE, Alan Conrad Bovik, Hamid Rahim Sheikh, , and Eero P. Simoncelli, Image Quality Assessment: From Error Visibility to Structural Similarity, IEEE Transactions On Image Processing, Vol. 13, No. 4, April 2004. https://doi.org/10.1109/TIP.2003.819861

20. M. Prema Kumar and P. Rajesh Kumar, A Multi Sensor Monochrome Video Fusion using Image Quality Assessment, International Conference on Communication and Network Technologies (ICCNT), 2014. https://doi.org/10.1109/CNT.2014.7062772

21. Megan Soni, Asst. Prof. Anand Khare and Asst. Prof Saurab Jain, Survay of Digital Image Processing and Its Problem, 2014.
22. Frances Bodrucki, Andrew Hill, Justin Davis, and John Cordell, Optical Modulation and Antialiasing Methods for Experimental Verification of Sensor Signal Integrity, IEEE Transactions On Instrumentation And Measurement, Vol. 67, No. 1, January 2018. https://doi.org/10.1109/TIM.2017.2753358

23. Pablo Arbela 'ez, Michael Maire, Charless Fowlkes, Member and Jitendra Malik, Contour Detection and Hierarchical Image Segmentation, IEEE Transactions On Pattern Analysis And Machine Intelligence, Vol. 33, No. 5, May 2011. https://doi.org/10.1109/TPAMI.2010.161

24. Ajoy K.Ray and Tinku Acharya, Image Processing Principle and Fundamental, A John Wiley \& Sons, $M c$, Publication, 2018

25. O. Saint-Pe', P. Magnan, M. Bre'art de Boisanger, M. Tulet, R. Davancens, P. Martin Gonthier, F. Corbie re, and N.Hunger, High-performance imagers for space applications: The strong benefits of CMOS image sensors processes, Nuclear Instruments and Methods in Physics Research, 2010 https://doi.org/10.1016/j.nima.2009.05.087 